\title{
СТИГМА КАК ФАКТОР ПСИХОГЕННОЙ ДЕПРЕССИИ У ПОДРОСТКОВ С ГОМОСЕКСУАЛЬНОСТЬЮ И БИСЕКСУАЛЬНОСТЬЮ
}

\begin{abstract}
Аннотация. Подростковый возраст как период формирования сексуальной идентичности можно назвать одним из наиболее сложных этапов становления личности, сопряженных со множеством трудностей, возникающих в таких срерах как межличностное взаимодействие и самоотношение. Поскольку во многих современных странах, в том числе, и в России, общественные установки и воззрения до сих пор патологизируют и криминализируют девиантное сексуальное влечение, подростки с дирфрузной половой идентичностью, а также те, кто осознанно относит себя к гомо- и бисексуалам, нередко вынуждены сталкиваться с непониманием, неприятием, дискриминацией и даже прямой травлей со стороны социального окружения. По данным литературы, большинство таких подростков подвергаются стигматизации, которая закономерно приводит к таким негативным последствиям, как психогенные депрессивные состояния, тревожные и другие стрессовые расстройства; резко повышается риск аутоагрессивного и суицидального поведения, наркотизации и алкоголизации, виктимизации. Цель данной работы: изучить роль стигмы в возникновении и выраженности психогенной депрессии у подростков с гомо- и бисексуальностью. Было обследовано 70 подростков с гомосексуальной и бисексуальной ориентацией в возрасте 12-16 лет. Психодиагностический инструментарий включал в себя шкалу А. Кинси, разработанную авторами короткую анкету для оценивания воспринимаемой стигмы, шкалу депрессии А.Т. Бека. Результаты исследования показали, что 96\% обследованных подростков подвергались стигматизации из-за их сексуальной ориентации. Было обнаружено, что более половины обследованных респондентов страдают от выраженных 8 различной степени симптомов депрессии. При этом стигматизация, как показал анализ, играет значимую роль в возникновении депрессивных расстройств психогенного характера у данного контингента лиц. Перспективу применения результатов исследования авторы видят в последующей разработке/модификации моделей психотерапии и психосоциальной поддержки для молодых людей, страдающих невротических и аффрективных расстройств, обусловленных стигматизацией и дискриминацией на почве ненависти к лицам с квир-гендерной идентичностью.
\end{abstract}

Ключевые слова: Бисексуальность, Гомосексуальность, Сексуальная ориентация, Подростки, Стрессовые расстройства, Депрессия, Дискриминация, Стигматизация, Буллинг, Структурное моделирование.

Abstract. As a period when sexual identity is formed, teenage years can be considered to be one of the most difficult stages of personality development associated with numerous difficulties arising in such spheres as interpersonal relationships and self-conception. Providing that in many modern countries including Russia social attitudes and public opinions pathologize deviant sexual desire, teenagers with diffusive sexual identity and those who recognize themselves as homoor bisexual often face misunderstanding, resentment and even bullying by their social surroundings. As the sources report, the majority of such teenagers are also go through stigmatization. This naturally leads to such negative consequences as psychogenic depression, anxiety and other stress disorders, drastically raise the risk of self-injurious and suicidal behavior, narcotization, alcohol addiction and victimization. The purpose of the present research is to study the role of stigma in the development and severity of psychogenic depression experienced by homosexual and bisexual teenagers. 70 homosexual and bisexual teenagers aged 12-16 years participated in the research. Teenagers took the following psychological tests: the Kinsey scale, the Beck Depression Inventory and a brief questionnaire prepared by the authors of the research to evaluate the level of stigmatization experienced by teenagers. According to the results, 96 percent of teenagers participating in the research have experienced stigmatization because of their sexual orientation. The research

Публикация подготовлена в рамках поддержанного РГНФ научного проекта №16-36-01050. 


\section{Психология и психотехника 8(95) • 2016}

also demonstrate that more than half of the respondents suffer from different degrees of depressive symptoms. Based on the results of data analysis, stigmatization plays an important role in the development of psychogenic depression for this group of teenagers. The results of the research can be used for further development/modification of models and methods used in psychotherapy and psychosocial support of young people who suffer from neurosis and mood disorders because of stigmatization and discrimination of people with queer-gender identity.

Key words: stigmatization, discrimination, depression, stress disorder, teenagers, sexual identity, homosexuality, bisexuality, bullying, structure modeling.

\section{Введение}

В конце XIX века проблема гомосексуальности человека во многих странах стала объектом науки. В России данная тема долгое время табуирована лишь с середины 1980-хх годов тема девиантной сексуальной ориентации стала доступна для обсуждения.

В настоящее время принято считать, что сексуальность человека определяется биологическими, социальными и психологическими детерминантами. Можно выделить как минимум три позиции к определению «рамок нормальности» сексуального поведения человека [1]. Позиция индивидуальной, физиологической нормы рассматривает гомосексуальность и бисексуальность, как ненормальные явления, т.к. гомосексуальный половой акт не способен привести к зачатию. Согласно позиции партнерской нормы, гомосексуальность может быть нормой, если оба партнера зрелые, стремятся к обоюдному удовлетворению и не причиняют ущерба здоровью друг друга и другим людям. При этом критерий различия пола в рамках этого подхода не позволяет считать гомосексуальность одним из вариантов нормы. И последняя - позиция правовой нормы рассматривает гомосексуальность нормальной в тех случаях, когда законодательство государства, гражданами которого являются люди с гомосексуальной ориентацией, не рассматривает гомосексуальность как правонарушение.

На сегодняшний день известно, что до 6-17\% всех подростков в определённый период их жизни испытывали сексуальное влечение к лицам того же пола [2].

Ученые полагают [3], что примерно 64\% гомосексуальных подростков подвергаются травле среди сверстников. Школьный буллинг, включая вербальную агрессию и насилие, для многих гомосексуальных подростков является довольно распространенным явлением. Это может сильно осложняться негативным отношением и порицанием со стороны родителей и иных близких [4]. Swearer et al. (2008) показал, что наиболее распространенными формами школьной дискриминации ЛГБТподростков являются издевки, причинение ущерба имуществу, социальная изоляция и физическое насилие. Самым частым типом гомофобного поведе- ния является навешивание оскорбительных ярлыков на гомосексуальных и бисексуальных лиц [5].

Гомосексуальность не служит основанием для возникновения депрессии - тем не менее, непризнание со стороны других людей и общественная дискриминация, которой подвергаются люди с гомосексуальной ориентацией, нередко приводит к возникновению депрессивных симптомов. Значительное число гомосексуалов ощущает страх обращения за специализированной медицинской помощью, что может способствовать ухудшению состояния здоровья. В результате большинство подростков с гомосексуальной и бисексуальной ориентацией страдают от симптомов психогенной депрессии и невротических расстройств $[6,7,8]$.

Подростки, которые идентифицируют себя с геями, лесбиянками или бисексуалами, в 2-7 раз чаще склонны к суицидальным мыслям и попыткам. Самый высокий риск суицидального поведения наблюдается в тех случаях, когда подросток осознает гомосексуальную идентичность в более раннем возрасте, когда есть семейные конфликты, вынуждающие его сбегать из дома в связи с тем, что его сексуальная ориентация противоречит семейным ценностям и установкам, а также если подросток не может поделиться своими проблемами с кем-то еще, кроме родителей. Эмпирических данных по завершенным суицидам не так много, но есть доказательства того, что непропорционально большое число самоубийств совершается именно среди гомо- и бисексуалов [9].

Большая доказательная база свидетельствует о том, что ЛГБТ-люди испытывают более высокий уровень депрессии и тревоги $[10,11,12,13,14]$.

Что касается влияния отдельных типов стигматизации на здоровье гомосексуалов, показано, что реализованная стигма тесно коррелирует с суицидальными мыслями [15], [16], эмоциональным дистрессом [17], проблемами психического здоровья [18].

\section{Материалы и методы}

Выборка исследования состояла из 70 подростков в возрасте 12-16 лет (средний возраст - 14,05 лет), которые имели сексуальное/эротическое влечение 


\section{Клиническая психология}

к лицам того же пола либо относили себя к гомосексуалам/бисексуалам. Формирование выборки проходило посредством крупнейшего российского сообщества в известной социальной сети, озаглавленного как «Дети-404».

Mетоды: стигматизация и буллинг оценивались с помощью разработанной авторами статьи короткой анкеты, которая включала в себя такие пункты, как отношение родителей к гомосексуальной ориентации их детей, вербальная агрессия в школе среди сверстников, а также насилие как минимум один раз на почве ненависти к гендерквиру. Выраженность стигмы оценивалась в зависимости от ответов респондентов в диапазоне от 0 (отсутствие стигматизации) до 3 (самая тяжелая степень стигматизации). Выраженность гомосексуального влечения оценивалась с помощью Шкалы Кинси. Эта методика использовалась с целью описания сексуального опыта человека и реакций на момент обследования. Уровень депрессии оценивался по шкале депрессии А.Т. Бека. Для статической обработки данных использовалось структурное моделирование. Расчеты проводились в программе IBMSPSSStatistics 22 с использованием программного пакета Amos.

\section{Результаты и обсуждение}

Описательные статистики показали, что 51 (72,9\%) обследованный подросток имел хотя бы один половой акт с лицом того же пола. Остальные 19 $(27,1 \%)$ не имели гомосексуального опыта, однако, испытывали стойкое влечение к людям, имеющим такой же пол. Шкала Кинси позволила установить, что 14 (20\%) респондентов были исключительно гомосексуальными, 3 (4,3\%) подростка были преимущественно гомосексуальны, лишь случайно гетеросексуальными, 5 (7,1\%) подростков были преимущественно гомосексуальны, но больше, чем случайно гетеросексуальными, 42 (60\%) участника были бисексуальными (одинаково гетеросексуальными и гомосексуальными). 48 (68,6\%) девушек были отнесены к бисексуалам, 11 (15,7\%) были лесбиянками. 4 (5,7\%) мальчиков были бисексуальны, в то время как остальные 7 (10\%) были исключительно гомосексуальны.

Как показало структурированное интервью на основе короткой анкеты, 67 (95,7\%) подростков подвергались стигматизации и буллингу. Только 3 (4,3\%) участника ответили, что они никогда не испытывали влияние стигмы. Все 67 респондентов сказали, что чувствовали нейтральное или негативное (более часто) отношение родителей к их сексуальной ориентации. 49 (70\%) подрост-

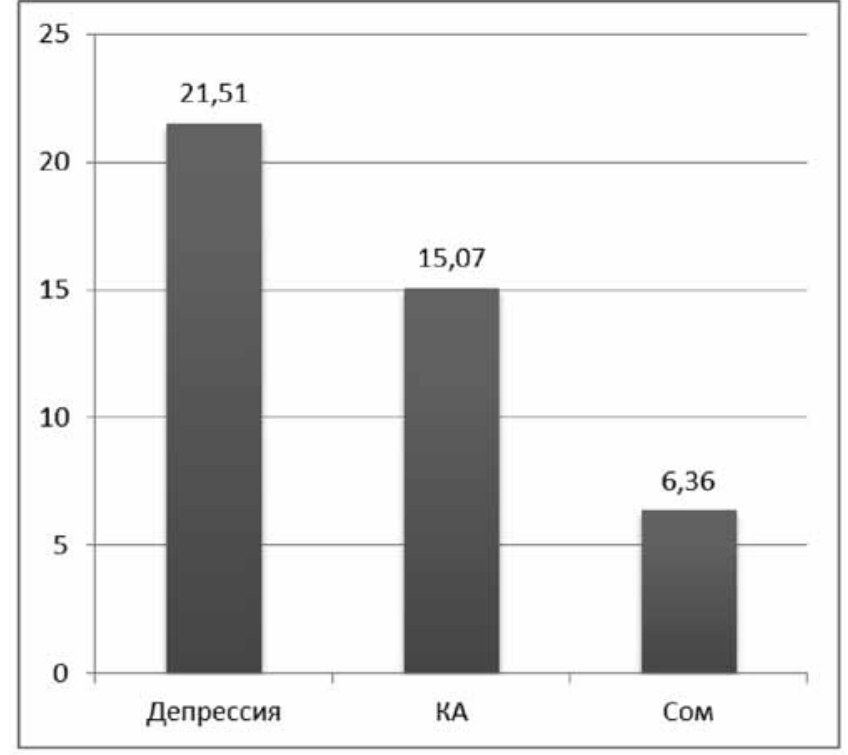

Рис. 1. Средние показатели выраженности депрессии по шкале А. Бека

Примечание: КА - когнитивно-аффективная субшкала; Сом - субшкала соматических проявлений депрессии.

ков сообщили, что они стали жертвами вербальной агрессии (ругательства, навешивание ярлыков, насмешки) со стороны сверстников в школе. 24 (34,3\%) участника признались, что были хотя бы раз побиты одноклассниками из-за их гомосексуальности. При этом $8(11,4 \%)$ человек ответили, что они подвергаются физическому насилию регулярно.

Что касается уровня выраженности депрессивных симптомов, было выявлено, что 14 (20\%) подростков имели легкую степень депрессии по шкале Бека $[19,20] .7$ (10\%) респондентов имели умеренный уровень депрессии. Тяжелая депрессия была выявлена у 19 (27,2\%) человек. Общие результаты исследования депрессии представлены на рис.1.

Как видно из представленной диаграммы, в целом для группы гомосексуальных подростков характерна высокая степень выраженности депрессивных проявлений. Для сравнения, в норме по шкале Бека показатели депрессии у подростков не должны быть выше 9 баллов. Установленный нами уровень депрессии свидетельствует о том, что в большинстве случаев у подростков уже на момент исследования имеются клинические проявления эмоциональных расстройств, требующие клиникопсихологического вмешательства.

Как показало структурное моделирование (рис. 2), стигматизация и школьный буллинг прямо влияют на уровень депрессии у гомосексуальных и бисексуальных подростков. 


\section{Психология и психотехника 8(95) • 2016}

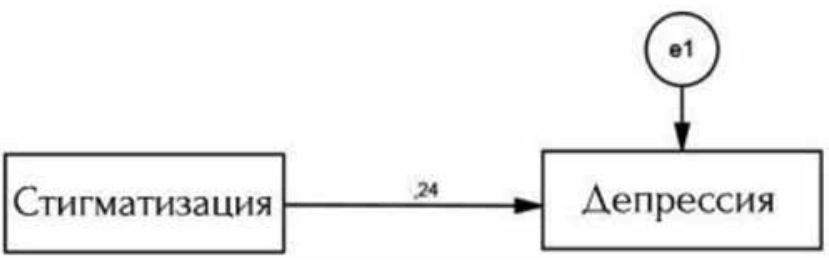

Рис. 2. Модель влияния стигматизации на депрессию у гомосексуальных подростков

Как оказалось, существенная доля факторов, которые влияют на возникновение депрессивных симптомов у гомосексуальных подростков, включает в себя негативное отношение общества к проявлениям гомосексуальности.

\section{Выводы}

Исследование показало, что 67 из 70 обследованных подростков подвергались стигматизации и буллингу из-за их сексуальной ориентации. Ругательства, насмешки, обзывательства, предрассудки и стереотипы, школьное насилие - это основные проявления анти-гомосексуальной дискриминации, с которой сталкиваются подростки из секс-меньшинств. К этому можно добавить важ- нейший фактор, такой как негативное отношение и порицание их родителей.

Мы обнаружили, что более половины обследованных респондентов страдают легкой, умеренной или тяжелой формами психогенной депрессии. При этом стигматизация и буллинг играют существенную роль в возникновении стрессовых и аффективных расстройств психогенного характера, которые могут проявляться на эмоциональном и соматическом уровнях. Вероятно, страх раскрытия сексуальной ориентации, низкая самооценка, трудности обучения и повседневные проблемы могут также вносить вклад в появление депрессивных симптомов, тем не менее, в рамках данного исследования эти детерминанты нами не учитывались.

Перспектива этого исследования предполагает дальнейшее изучение личностных ресурсов и когнитивных стилей, помогающих преодолевать влияния стигмы и стрессов у подростков с гомосексуальностью и бисексуальностью. Авторы видят продолжение этого исследования в аспекте разработки/модификации существующих моделей психотерапии и психосоциальной поддержки для молодых людей, страдающих от депрессии в связи с их сексуальной ориентацией.

\section{Список литературы:}

1. Ерзин А.И. Сексуальность в норме и патологии. - Palmarium Academic Publishing. 2013. - 265 c.

2. American Academy of Pediatrics, Committee on Adolescence. (1993). Homosexuality and Adolescence. Pediatrics, 92, 631634. Retrieved from http://pediatrics.aappublications.org/content/pediatrics/92/4/631.full.pdf

3. Johnson R. M., Kidd J. D., Dunn E. C., Green J. G., Corliss H. L., Bowen D. Associations Between Caregiver Support, Bullying, and Depressive Symptomatology Among Sexual Minority and Heterosexual Girls: Results from the 2008 Boston Youth Survey. J Sch Violence. Author manuscript; available in PMC 2012 June 14. Published in final edited form as: J Sch Violence. 2011; 10(2): 185-200. doi: 10.1080/15388220.2010.539168

4. Tritt R.J. Attitudes of adults towards homosexuality and knowledge of sexually transmitted diseases: part one. Ann Acad Med Stetin. 2011;57(2):46-56.

5. Rivers. I. Social exclusion. absenteeism and sexual minority youth // Support for Learning.-2000.-Vol. 15(1).-P. 13-18.

6. Alessi E. J., Martin J. I., Gyamerah A., Meyer I. H. Prejudice-Related Events and Traumatic Stress Among Heterosexuals and Lesbians, Gay Men and Bisexuals. J Aggress Maltreat Trauma. Author manuscript; available in PMC 2014 January 1. Published in final edited form as: J Aggress Maltreat Trauma. 2013 January 1; 22(5): 510-526. Published online 2013 May 17.

7. Barnard A. Lesbians' constructions of depression. Health Care Women Int. 2009 May;30(5):373-89. doi: 10.1080/07399330902785141.

8. Shenkman G., Shmotkin D. Mental health among Israeli homosexual adolescents and young adults. J Homosex. 2011;58(1):97116. doi: 10.1080/00918369.2011.533630.).

9. Remafedi G. Sexual orientation and youth suicide // JAMA.-1999.-Vol. 282.-P.1291-2.

10. King et al. (1988). Canada. Youth and AIDS Study. Kingston. ON: Queen's University.

11. Brennan DJ., Dobinson C., Ross LE., Steele LS., Veldhuizen S. Men's sexual orientation and health in Canada // Can J Public Health.-2010.-Vol. 101.-P. 255-258.

12. Cochran S.D., Mays V.M. Burden of psychiatric morbidity among lesbian, gay and bisexual individuals in the California Quality of Life Survey // J Abnorm Psychol.-2009.-Vol. 118.-P. 647-658. doi: 10.1037/a0016501 PMID: 19685960

13. Frisell T., Langstrom N., Lichtenstein P., Rahman Q. Psychiatric morbidity associated with samesex sexual behaviour: influence of minority stress and familial factors // Psychol Med.-2010.-Vol. 40.-P. 315-324.-doi: 10.1017/S0033291709005996 PMID: 19460186

14. Lewis N.M. Mental health in sexual minorities: recent indicators, trends and their relationship to place in North America and Europe // Health Place.-2009.-Vol. 15.-P. 1029-1045.-doi: 10.1016/j.healthplace.2009.05.003 
15. Duncan D.T., Hatzenbuehler M.L. Lesbian, Gay, bisexual and transgender hate crimes and suicidality among a populationbased sample of sexual-minority adolescents in Boston // Am J Public Health.-2014.-Vol. 104.-P. 272-278.-doi: 10.2105/ AJPH.2013.301424 PMID: 24328619

16. Liu RT., Mustanski BSuicidal ideation and self-harm in lesbian, gay, bisexual and transgender youth. // Am J Prev Med.-2012.Vol. 42.-P. 221-228.-doi: 10.1016/j.amepre.2011.10.023 PMID: 22341158

17. D'Augelli AR., Herschberger SL, Pilkington NW. Incidence and mental health impact of sexual orientation victimization of lesbian. gay and bisexual youths in high school // School Psychol Quart.-2002.-Vol. 17.-P. 148-167.

18. Kuyper L., Vanwesenbeeck I Examining sexual health differences between lesbian, gay, bisexual and heterosexual adults: the role of sociodemographics, sexual behavior characteristics and minority stress // J Sex Res 48.-2011.-P. 263-274.-doi: $10.1080 / 00224491003654473$

19. Beck, A. T., Ward, C. H., Mendelson, M., Mock, J., \& Erbaugh, J. (1961). An inventory for measuring depression. Archives of General Psychiatry, 4, 561-571.

20. Beck, A. T., Steer, R. A., \& Garbin, M. G. (1988). Psychometric properties of the Beck Depression Inventory: twenty-five years of evaluation. Clinical Psychology Review, 8(1), 77-100.

\section{References (transliterated):}

1. Erzin A.I. Seksual'nost'v norme i patologii. - Palmarium Academic Publishing. 2013. - 265 s.

2. American Academy of Pediatrics, Committee on Adolescence. (1993). Homosexuality and Adolescence. Pediatrics, 92, 631634. Retrieved from http://pediatrics.aappublications.org/content/pediatrics/92/4/631.full.pdf

3. Johnson R. M., Kidd J. D., Dunn E. C., Green J. G., Corliss H. L., Bowen D. Associations Between Caregiver Support, Bullying, and Depressive Symptomatology Among Sexual Minority and Heterosexual Girls: Results from the 2008 Boston Youth Survey. J Sch Violence. Author manuscript; available in PMC 2012 June 14. Published in final edited form as: J Sch Violence. 2011; 10(2): 185-200. doi: 10.1080/15388220.2010.539168

4. Tritt R.J. Attitudes of adults towards homosexuality and knowledge of sexually transmitted diseases: part one. Ann Acad Med Stetin. 2011;57(2):46-56.

5. Rivers. I. Social exclusion. absenteeism and sexual minority youth // Support for Learning.-2000.-Vol. 15(1).-P. 13-18.

6. Alessi E. J., Martin J. I., Gyamerah A., Meyer I. H. Prejudice-Related Events and Traumatic Stress Among Heterosexuals and Lesbians, Gay Men and Bisexuals. J Aggress Maltreat Trauma. Author manuscript; available in PMC 2014 January 1. Published in final edited form as: J Aggress Maltreat Trauma. 2013 January 1; 22(5): 510-526. Published online 2013 May 17.

7. Barnard A. Lesbians' constructions of depression. Health Care Women Int. 2009 May;30(5):373-89. doi: $10.1080 / 07399330902785141$.

8. Shenkman G., Shmotkin D. Mental health among Israeli homosexual adolescents and young adults. J Homosex. 2011;58(1):97116. doi: 10.1080/00918369.2011.533630.).

9. Remafedi G. Sexual orientation and youth suicide // JAMA.-1999.-Vol. 282.-P.1291-2.

10. King et al. (1988). Canada. Youth and AIDS Study. Kingston. ON: Queen's University.

11. Brennan DJ., Dobinson C., Ross LE., Steele LS., Veldhuizen S. Men's sexual orientation and health in Canada // Can J Public Health.-2010.-Vol. 101.-P. 255-258.

12. Cochran S.D., Mays V.M. Burden of psychiatric morbidity among lesbian, gay and bisexual individuals in the California Quality of Life Survey // J Abnorm Psychol.-2009.-Vol. 118.-P. 647-658. doi: 10.1037/a0016501 PMID: 19685960

13. Frisell T., Langstrom N., Lichtenstein P., Rahman Q. Psychiatric morbidity associated with samesex sexual behaviour: influence of minority stress and familial factors // Psychol Med.-2010.-Vol. 40.-P. 315-324.-doi: 10.1017/S0033291709005996 PMID: 19460186

14. Lewis N.M. Mental health in sexual minorities: recent indicators, trends and their relationship to place in North America and Europe // Health Place.-2009.-Vol. 15.-P. 1029-1045.-doi: 10.1016/j.healthplace.2009.05.003

15. Duncan D.T., Hatzenbuehler M.L. Lesbian, Gay, bisexual and transgender hate crimes and suicidality among a populationbased sample of sexual-minority adolescents in Boston // Am J Public Health.-2014.-Vol. 104.-P. 272-278.-doi: 10.2105/ AJPH.2013.301424 PMID: 24328619

16. Liu RT., Mustanski BSuicidal ideation and self-harm in lesbian, gay, bisexual and transgender youth. // Am J Prev Med.-2012.Vol. 42.-P. 221-228.-doi: 10.1016/j.amepre.2011.10.023 PMID: 22341158

17. D'Augelli AR., Herschberger SL, Pilkington NW. Incidence and mental health impact of sexual orientation victimization of lesbian. gay and bisexual youths in high school // School Psychol Quart.-2002.-Vol. 17.-P. 148-167.

18. Kuyper L., Vanwesenbeeck I Examining sexual health differences between lesbian, gay, bisexual and heterosexual adults: the role of sociodemographics, sexual behavior characteristics and minority stress // J Sex Res 48.-2011.-P. 263-274.-doi: $10.1080 / 00224491003654473$

19. Beck, A. T., Ward, C. H., Mendelson, M., Mock, J., \& Erbaugh, J. (1961). An inventory for measuring depression. Archives of General Psychiatry, 4, 561-571.

20. Beck, A. T., Steer, R. A., \& Garbin, M. G. (1988). Psychometric properties of the Beck Depression Inventory: twenty-five years of evaluation. Clinical Psychology Review, 8(1), 77-100. 